\title{
Anti-aging effects of extracts prepared from salmon nasal cartilage in hairless mice
}

\author{
MASASHI GOTO ${ }^{1,4}$, SEIKO ITO ${ }^{2}$, YOJI KATO ${ }^{2}$, SHOTA YAMAZAKI ${ }^{3}$, \\ KAZUSHI YAMAMOTO ${ }^{4}$ and YOHTARO KATAGATA ${ }^{1,3}$ \\ ${ }^{1}$ Science of Bioresources, The United Graduate School of Agricultural Sciences, Iwate University, \\ Morioka 020-8550; ${ }^{2}$ Laboratory of Food Science, Faculty of Education, Hirosaki University, \\ Hirosaki 036-8560; ${ }^{3}$ Department of Biochemistry and Biotechnology, Faculty of Agriculture and Life Science, \\ Hirosaki University, Hirosaki 036-8561; ${ }^{4}$ R\&D Department, Sunstar Inc., \\ Takatsuki 569-1195, Japan
}

Received March 8, 2011; Accepted May 17, 2011

DOI: $10.3892 / \mathrm{mmr} .2011 .498$

\begin{abstract}
The skin anti-aging effects of orally administered salmon nasal cartilage extract (SNCE), which includes abundant proteoglycan, were investigated using a hairless mouse skin-aging model, in which aging was caused by repetitive ultraviolet B (UV-B) irradiation. Physiological analysis of the skin surface following repetitive UV-B irradiation of 8 weeks revealed inhibition of erythema levels and reduction of transepidermal water loss (TEWL) due to oral administration of SNCE. Similarly, inhibitory actions of epidermal and dermal hypertrophy were revealed by hematoxylin and eosin staining. Furthermore, effects on the hydration level of the skin surface by SNCE administration were indicated at 4 weeks of UV-B irradiation, but greater effects were not apparent. These results indicate that SNCE may serve as an anti-aging agent for healthy skin.
\end{abstract}

\section{Introduction}

Dermis in the skin and cartilage are composed mainly of collagen, hyaluronic acid and proteoglycans, a family of complex macromolecules consisting of a core protein and covalently attached glycosaminoglycan (GAG) chains (1). Aggrecan, with the largest molecular mass among the proteoglycans, is abundant in cartilage and is composed of GAG, predominantly chondroitin sulfate (CS) (2). Vincent et al reported that proteoglycan of bovine nasal cartilage contains $87 \%$ CS, $6 \%$ keratan sulfate and $7 \%$ protein (3).

Correspondence to: Dr Yohtaro Katagata, Department of Biochemistry and Biotechnology, Faculty of Agriculture and Life Science, Hirosaki University, 3 Bunkyo-cho, Hirosaki 036-8561, Japan

E-mail:katagata@cc.hirosaki-u.ac.jp

Key words: proteoglycan, salmon nasal cartilage, anti-aging function
Previously, collagen and hyaluronic acid were extensively investigated with regard to oral intake and have become widely used as food and are also available in the form of supplements intended to improve beauty and health. Gelatin, which is denatured collagen, has elicited noteworthy data from animal experiments showing increased bone mineral density following oral intake $(4,5)$. Furthermore, the oral intake of collagen peptide suppressed UV-B-induced decreases in skin hydration and epidermal hypertrophy, and decreased soluble type I collagen in animal (hairless mice) experiments (6). The oral intake of hyaluronic acid has produced significant reductions of dry skin and skin roughness in human experiments (7,8). Despite numerous investigations into similar physiological effects, no study of proteoglycan by oral intake has as yet been reported, and no reports are currently available comparing the effects on skin with those of collagen and hyaluronic acid. Recently, Mitsui et al observed, using animal (rat) experimental systems, that oral administration of proteoglycan extracted from salmon nasal cartilage suppressed the progression of colitis (9).

The skin contacts the outer environment since it is an organ occurring predominantly outside of the human body. It is affected by photoaging from daily irradiation of UV-A and UV-B. Furthermore, skin reverts to a low level of epidermal barrier function when irradiated by UV and exhibits a notably increased transepidermal water loss (TEWL), which eventually induces dry skin $(10,11)$. Additionally, skin is reportedly stiffened by epidermal and dermal hypertrophy: wrinkles form as a result of photoaging (12). Using an experimental animal model established for photoaging, the present study was undertaken to determine whether oral administration of salmon nasal cartilage extract (SNCE), which contains high levels of proteoglycan, affects the recovery of hairless mouse skin irradiated by UV-B.

\section{Materials and methods}

Preparation of salmon nasal cartilage extract. Nasal cartilage was cut from the salmon head area and washed with water. The cartilage was crushed finely using a meat chopper, 
then suspended in 1-2 volumes of water and centrifuged at $8,000 \mathrm{rpm}$ for $30 \mathrm{~min}$ at room temperature. Residues were then collected. This procedure was performed twice. The obtained residues were lyophilized and powdered carefully. The powder was suspended in 10 volumes of ethanol, filtered using mesh and dried to obtain defatted powders, which were suspended at a concentration of $2 \%$ in water and centrifuged at $15,000 \mathrm{rpm}$ for $30 \mathrm{~min}$ at $4^{\circ} \mathrm{C}$. After centrifugation, the obtained supernatant was lyophilized. The SNCE was not treated with acid, alkaline or enzymolysis in order to avoid proteoglycan degradation.

Quantity of glucuronic acid. CS is the repeated sequence of D-glucuronic acid and N-acetyl-D-galactosamine bound by sulfuric acid. The glucuronic acid levels of SNCE and CS were quantified using the carbazole-sulfuric acid method, since it has been used as a general method to determine CS (13).

Gel filtration column chromatography. Water soluble extract (1 ml; $4 \mathrm{mg} / \mathrm{ml})$ in SNCE was applied to a Sepharose CL-2B column $(1.0 \phi \times 50 \mathrm{~cm})$ that had previously been equilibrated with $0.1 \mathrm{M}$ phosphate buffer ( $\mathrm{pH} 7.1$ ) containing $0.2 \mathrm{M}$ sodium chloride. It was eluted using the same buffer. Concentrations of glucuronic acid and protein in each fraction $(1 \mathrm{ml})$ were determined, respectively, using the carbazole-sulfuric acid method and absorbance at $280 \mathrm{~nm}$.

Chondroitin sulfate. CS was purchased as a commercial raw material (Sake PG-20; Maruha Nichiro Holdings Inc., Tokyo, Japan), which was obtained by enzymolysis of salmon nasal cartilage and included $50 \%$ dextrin.

Animals. Male hairless mice (Hr-/Kud), 5-6 weeks old, were purchased from Kyudo Co., Ltd. (Fukuoka, Japan). The animals were fed a standard diet and water ad libitum. The mice were housed 6 per cage and maintained at a constant temperature $\left(22 \pm 2^{\circ} \mathrm{C}\right)$ with a 12 -h light/dark cycle. Mice were 7 weeks old at the start of experimentation. Animal experiments in this study were carried out in accordance with the guidelines for animal experimentation of the Hirosaki University.

Oral administration. Each cage was randomly allocated to a sample. Oral administration of each sample was performed with 6 mice in each cage. Each sample administration was commenced 3 weeks prior to UVB irradiation and then continued for 11 weeks. Mice from the 3 SNCE groups were orally administered $0.5 \mathrm{ml}$ of distilled water, which was dissolved in $3.5,0.875$ or $0.175 \mathrm{mg}$ of SNCE/mouse/day. In addition, the CS group was orally administered $0.5 \mathrm{ml}$ of distilled water, which was dissolved in $7.0 \mathrm{mg}$ of CS/mouse/ day. Two control groups (non-irradiated and UV-B-irradiated) were administered $0.5 \mathrm{ml}$ of distilled water only. The prepared sample and control were administered to the mice 5 times/ week using a stomach sonde needle (1.2 ф x 50 mm).

$U V-B$ irradiation. A bank of six 436-mm UV-B lamps (GL15E; Sankyo Denki Co., Ltd., Japan) was used. During the period of UV-B radiation, the mice were housed in a stainless steel irradiation chamber. The UV-B lamp height was adjusted to deliver $1.0 \mathrm{~mW} / \mathrm{cm}^{2}$ at the dorsal surface of the mice in a stainless steel irradiation chamber. The time was adjusted to deliver the appropriate UV-B daily irradiance. The mice were exposed to UV-B radiation 5 times a week, starting with $60 \mathrm{~mJ} / \mathrm{cm}^{2}$ as the minimal edematous dose (MED) for the first week, followed sequentially by $120 \mathrm{~mJ} / \mathrm{cm}^{2}$ (7 weeks) to constitute a total dose of $4.5 \mathrm{~J} / \mathrm{cm}^{2}$ over the 8 -week period. The UV-B irradiance was measured using a radiometer with a radiometer sensor (UVP UVX $310 \mathrm{~nm}$; UVP, LLC, Upland, CA, USA).

Physiological analysis in surface skin. A MPA 5 analytical system and its probes (Courage + Khazaka Electronic $\mathrm{GmbH}$, Germany) were used. The erythema value in the dorsal skin surface of the hairless mice was measured using a Mexameter (MX18; Courage + Khazaka Electronic $\mathrm{GmbH})$. The TEWL $\left(\mathrm{g} / \mathrm{hm}^{2}\right)$ in the dorsal skin surface of hairless mice was measured using a Tewameter (TM300; Courage + Khazaka Electronic $\mathrm{GmbH}$ ) as described above. The hydration level in the dorsal skin surface of hairless mice was measured using a Corneometer (CM825; Courage + Khazaka Electronic $\mathrm{GmbH}$ ) as described above. Results are presented as the means \pm SEM. Statistical analyses were performed using the Student's t-test. $\mathrm{P}<0.05$ was considered to be statistically significant. Each experiment was repeated at least twice.

Histological analysis. Biopsies were obtained from the central dorsal skin of hairless mice 1 day after the final UV-B irradiation. Skin biopsy samples were fixed in $18.5 \%$ formalin and embedded in paraffin. Serial sections $(3 \mu \mathrm{m})$ were mounted onto silane-coated slide glasses (Matsunami Glass Ind. Ltd., Osaka, Japan). After skin sections were stained with hematoxylin and eosin (H\&E), microphotographs were taken. Epidermal and dermal thicknesses were measured in 10 places in the microphotography of skin sections. Results are shown as the means \pm SEM. Statistical analyses were performed using the Student's t-test. $\mathrm{P}<0.05$ was considered to be statistically significant. Each experiment was repeated at least twice.

\section{Results}

Quantity of glucuronic acid. The glucuronic acid level of SNCE and CS was quantified using carbazole-sulfuric acid methods as an index of CS contents. This quantification accounted for 20.6 and $12.8 \%$ of glucuronic acid in SNCE and $\mathrm{CS}$, respectively. The ratio of glucuronic acid used to account for CS by calculation of the molecular weight was approximately $38 \%$. Therefore, the CS levels were evaluated at approximately 54 and 34\% in SNCE and CS, respectively.

Gel filtration of the SNCE and CS on a Sepharose CL-2B column. SNCE and CS were applied to a Sepharose CL-2B column $(1 \phi \times 50 \mathrm{~cm})$ equilibrated with $0.1 \mathrm{M}$ phosphate buffer ( $\mathrm{pH}$ 7.1) containing 0.2 M sodium chloride. The elution profiles are shown in Fig. 1. The SNCE exhibited a combined peak (one main peak and a shouldered one) as glucuronic acid (Fig. 1A). SNCE main peak was near void volume. Therefore, it was indicated that SNCE has a notably heavy molecular 
A

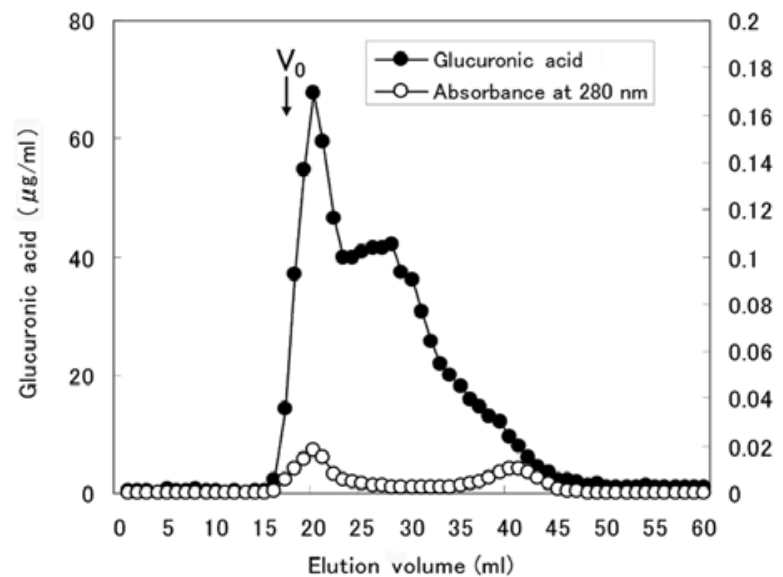

B

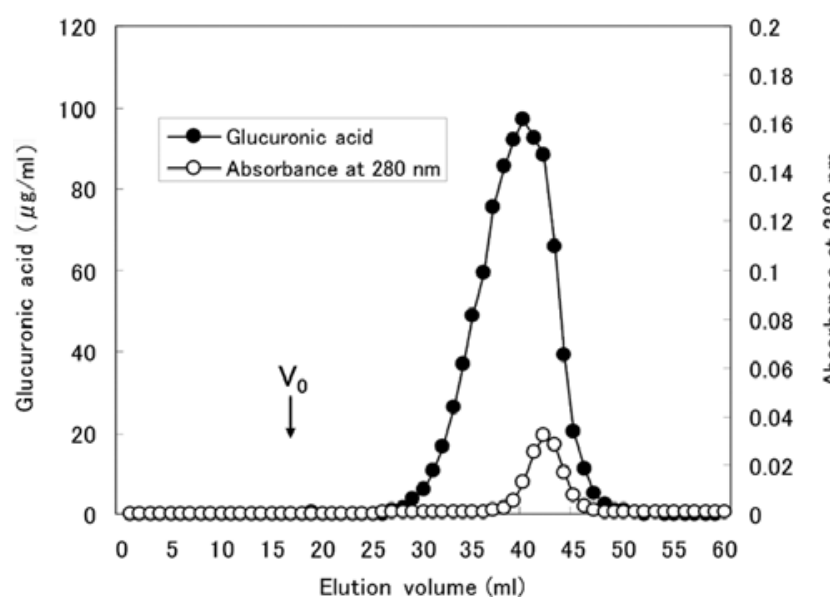

Figure 1. Sepharose CL-2B column chromatography of SNCE and CS. Supernatant $(1 \mathrm{ml})$ was applied to a Sepharose CL-2B column $(1.0 \times 50 \mathrm{~cm})$ equilibrated with $0.1 \mathrm{M}$ phosphate buffer ( $\mathrm{pH}$ 7.1) containing $0.2 \mathrm{M}$ sodium chloride. Aliquots $(0.1 \mathrm{ml})$ were removed from tubes and examined with the carbazole reaction. The flow rate was $9 \mathrm{ml} / \mathrm{h}$. (A) SNCE and (B) CS. weight. In contrast, $\mathrm{CS}$ exhibited one main peak that was isolated from void volume (Fig. 1B). Aggrecan, which is the largest molecular mass in proteoglycan, is abundant in cartilage (2). A comparison of the two elution profiles revealed that SNCE was detected at a larger position more easily than CS. SNCE was estimated as the presence of the original aggrecan form.

Physiological analysis of mouse skin surface by oral administration of SNCE and CS. Physiological aspects of mouse dorsal skin surface after oral administration of SNCE and CS were analyzed, based on data obtained after 4- and 8-week UV-B irradiation using an analytical system including a number of devices (MPA 5; Courage + Khazaka Electronic $\mathrm{GmbH}$ ) and their probes. Data of erythema values, TEWL and water contents of the stratum corneum are shown in Figs. 2-4, respectively. Fig. 2 shows that UV-B irradiation significantly increased the erythema value (non-irradiated and UV-B-irradiated control groups). However, the increase in the erythema value was found to be markedly decreased by the oral administration of SNCE (3.5 mg/mouse/day) and was diminished slightly with SNCE $(0.875 \mathrm{mg} / \mathrm{mouse} /$ day $)$ and CS (7 mg/mouse/day) after 4 weeks of UV-B irradiation. The phenomena described above were not clearly observed after 8 weeks, as shown in Fig. 2, except between non-irradiated and UV-B-irradiated control fractions. TEWL, which was increased by UV-B irradiation, was revealed as having been suppressed significantly by the data from the groups receiving SNCE administration at 4 and 8 weeks of UV-B irradiation (Fig. 3). In contrast, CS did not provide any data revealing a significant decrease in TEWL when administered at 4 or 8 weeks of UV-B irradiation. Fig. 4 shows that the hydration level, which was decreased by UV-B irradiation of the skin surface, was largely unaffected by SNCE administration for improvement of the mouse dorsal skin surface, with the exception of the fraction $(3.5 \mathrm{mg} / \mathrm{mouse} /$ day $)$ of SNCE administration.
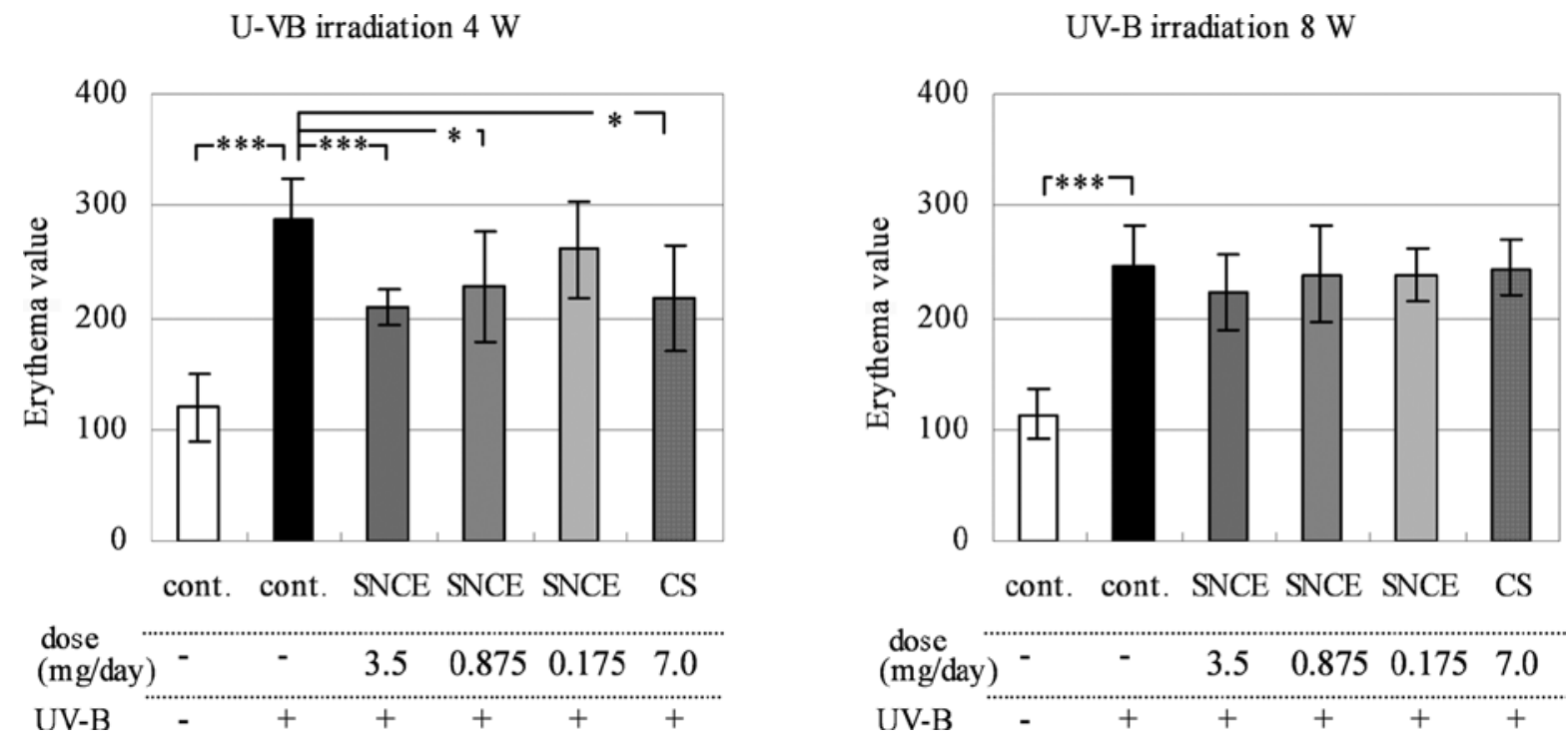

Figure 2. Expression levels of erythema values are indicated following oral administration of SNCE and CS in the dorsal skin surface of hairless mice. Values are the means \pm SEM. ${ }^{* * * *}$ p $<0.01$ vs. non-irradiated control group and ${ }^{*}$ p $<0.05$ vs. UV-B-irradiated control group. Cont., control; SNCE, salmon nasal cartilage extract; CS, chondroitin sulfate. 
UV-B irradiation $4 \mathrm{~W}$

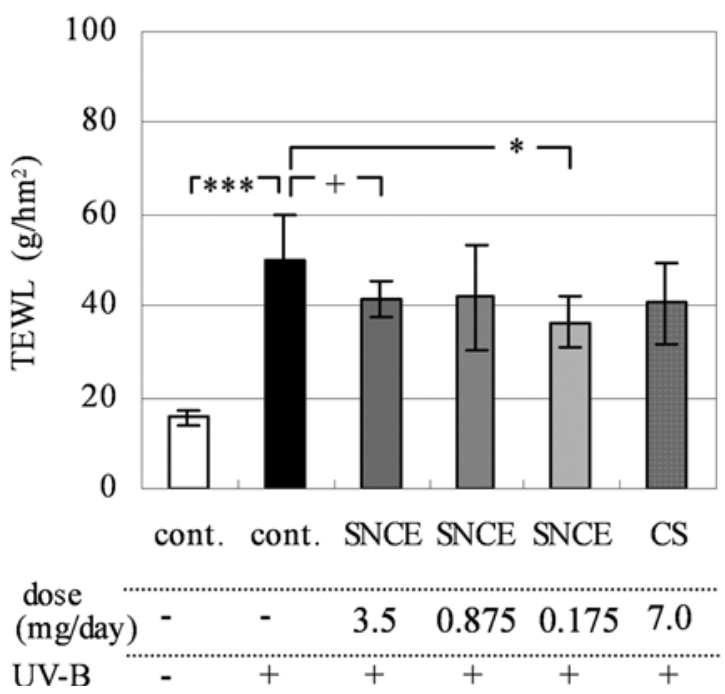

UV-B irradiation $8 \mathrm{~W}$

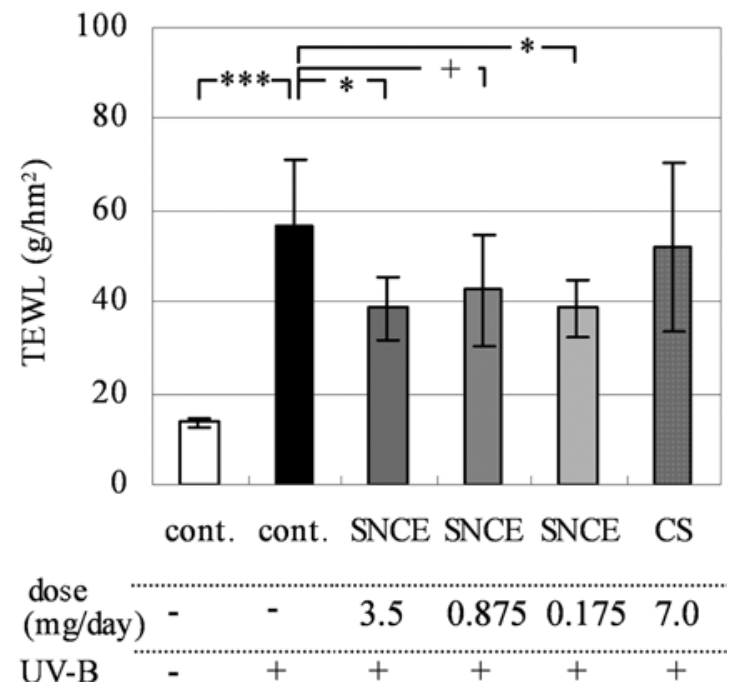

Figure 3. Expression levels of TEWL are shown following oral administration of SNCE and CS in a dorsal skin surface of hairless mice. Values are the means \pm SEM. ${ }^{* * * *}$ p $<0.01$ vs. non-irradiated control group and "p $<0.05$ vs. UV-B-irradiated control group. Cont., control; SNCE, salmon nasal cartilage extract; CS, chondroitin sulfate.
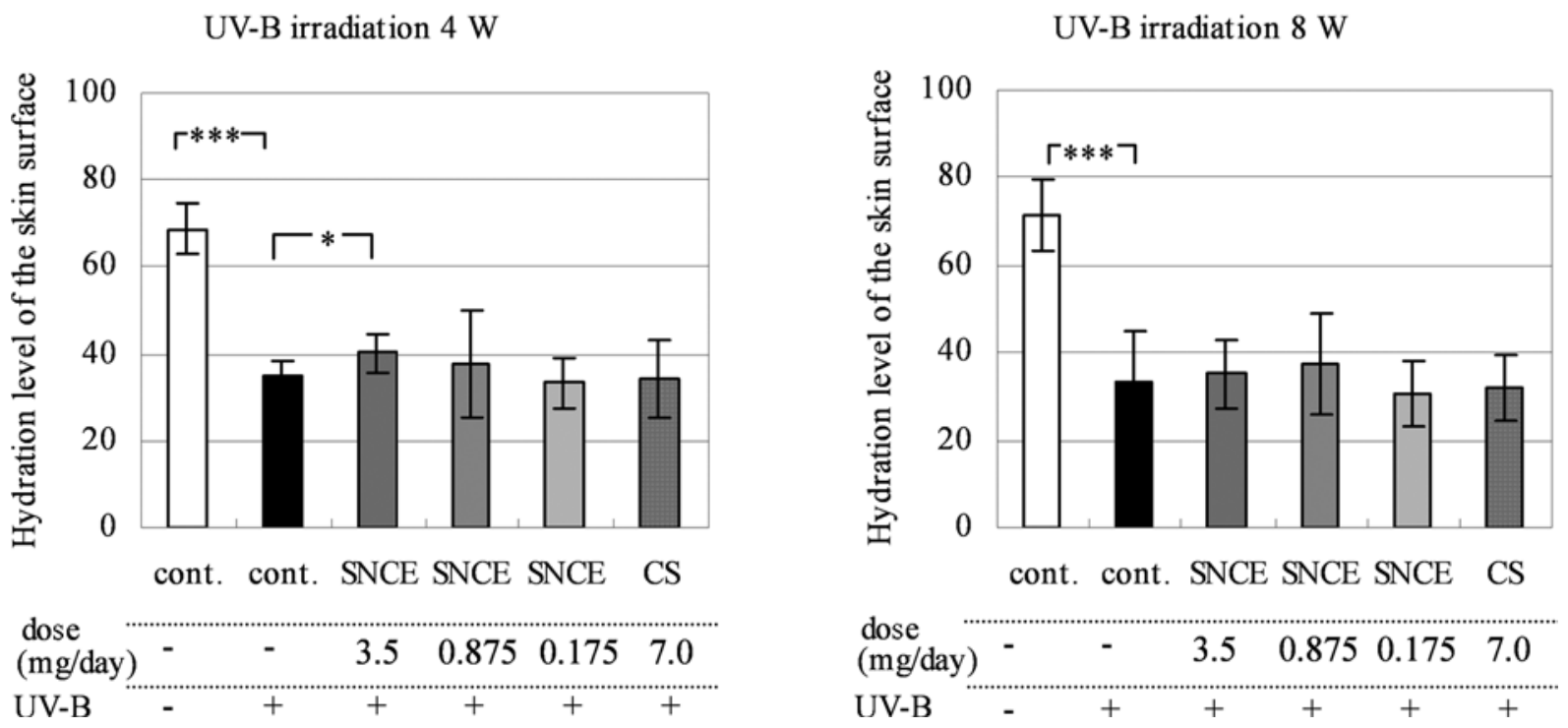

Figure 4. Hydration level of the skin surface is shown following oral administration of SNCE and CS on a hairless mouse dorsal skin surface. Values are the means \pm SEM. ${ }^{* * *} \mathrm{p}<0.01$ vs. non-irradiated control group and ${ }^{*} \mathrm{p}<0.05$ vs. UV-B-irradiated control group. Cont., control; SNCE, salmon nasal cartilage extract; CS, chondroitin sulfate.

Histological analysis. To demonstrate the physiological effects on mouse dorsal skin of orally administered SNCE and 8 weeks of UV-B irradiation, skin sections were stained using H\&E. Sections were photographed using a microscope (Fig. 5). For 8 weeks, UV-B irradiation produced epidermal and dermal hypertrophy (Fig. 5A vs. B). Administration of SNCE appeared to suppress epidermal and dermal thickness dose-dependently compared with the controls (Figs. 5B vs. C-E). The thickness of the two tissues was measured from the stained images (Fig. 6). Evidence of suppression in the two tissues was also observed. However, CS exhibited almost no effect in improving hypertrophy by oral administration.

\section{Discussion}

Judgement systems, which were affected sensitively by irritants of hairless mouse dorsal skin such as UV-B irradiation, were used as a skin aging model since the degree of effectiveness of SNCE administration alone was not expected to be significant. The UV-B irradiation was repeated 5 times per week for 8 weeks. In terms of appearance and texture, the hairless mouse dorsal skin produced erythema and was tightened by accumulated UV-B irradiation (total dose of $4.5 \mathrm{~J} / \mathrm{cm}^{2}$ ). CS, which was obtained by enzymolysis of the salmon nasal cartilage, was used as a control sample. In other words, CS was the resolution product of SNCE. 

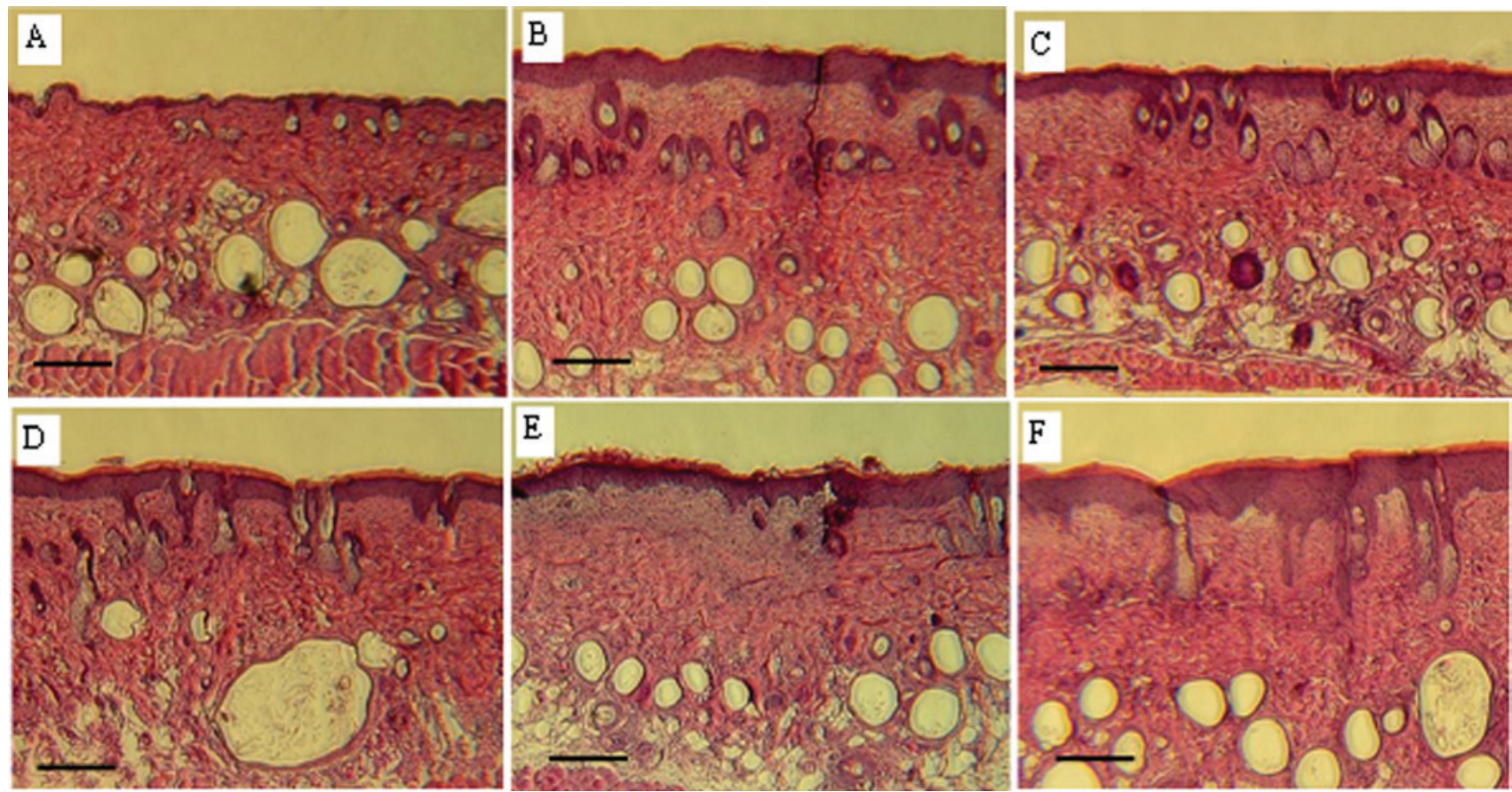

Figure 5. Hematoxylin and eosin (H\&E) staining data are shown in the dorsal skin of hairless mice following the oral administration of SNCE and CS. (A) non-irradiated control, (B) UV-B-irradiated control, (C) UV-B-irradiated and SNCE-administered (3.5 mg/ml), (D) UV-B-irradiated and SNCEadministered $(0.875 \mathrm{mg} / \mathrm{ml})$, (E) UV-B-irradiated and SNCE-administered $(0.175 \mathrm{mg} / \mathrm{ml})$ and (F) UV-B-irradiated and CS-administered (7.0 mg/ml). Scale bar, $200 \mu \mathrm{m}$. Magnification, $\mathrm{x} 40$.

Thickness of the epidermis
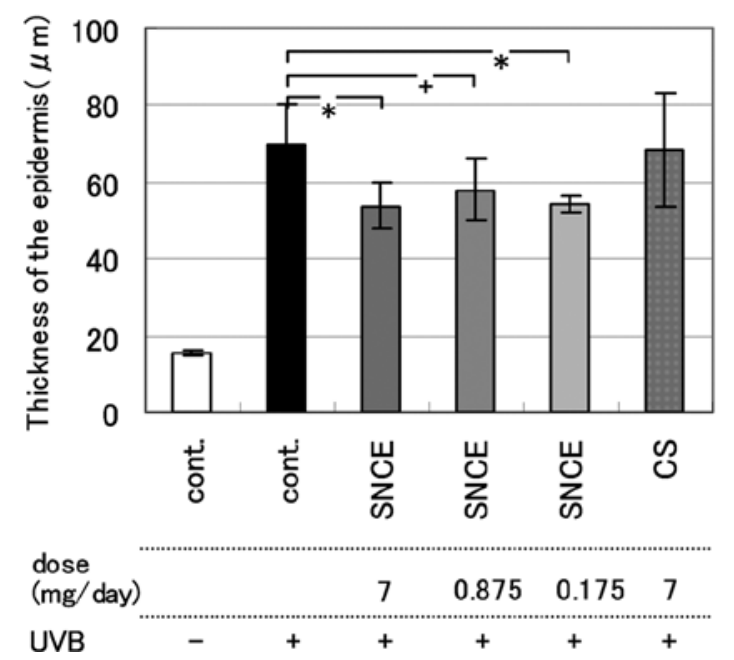

Thickness of the dermis

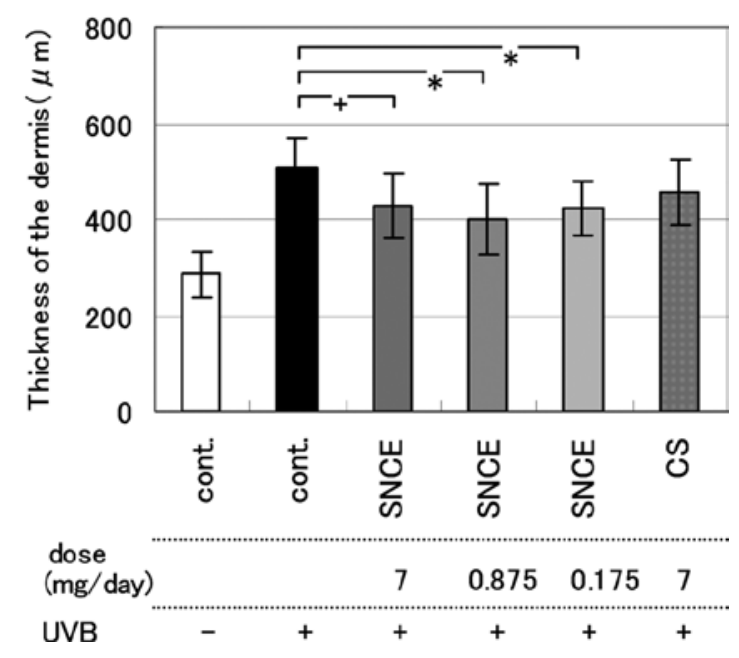

Figure 6. Epidermal and dermal thickness in the dorsal skin surface of hairless mice were measured following oral administration of SNCE and CS. These data were calculated from data presented in Fig. 5. Values are the means \pm SEM. ${ }^{*}<<0.05$ vs. UV-B-treated mice. Cont., control; SNCE, salmon nasal cartilage extract; CS, chondroitin sulfate.

Oral administration of SNCE mitigated various photoaging effects of UV-B irradiation, as shown in Figs. 1-5. These mitigated effects suppressed increased erythema level, increased TEWL, decreased hydration level of the skin surface, and epidermal and dermal hypertrophy. In other words, SNCE appeared to suppress inflammation, restrain erythema levels, control skin hypertrophy and maintain skin flexibility. The TEWL values can be regarded as a marker of the stratum corneum barrier functions $(14,15)$. Additionally, administration of TEWL may be regarded as protection against degradation in the epidermal barrier and as a preventative measure against dry skin. The mentioned results above show that oral administration of SNCE is effective for protection against photoaging.

We confirmed by measurement of glucuronic acid and gel filtration chromatography that aggrecan, a proteoglycan 
with large molecular mass, is abundant in SNCE. The SNCE used for this study is an extract obtained after crushing, lipid removal, and desiccation of salmon nasal cartilage. No operation was performed in the manufacturing process by which any acid, alkali, or enzyme was capable of resolving proteoglycan. Therefore, it appears that aggrecan remained in its original form. Aggrecan, which is present abundantly in salmon nasal cartilage, exhibited anti-aging effects on skin.

Reportedly, collagen is resolved into dipeptide (ProHyp) by digestive organs, after which it is absorbed, causing physiological activity $(16,17)$. However, it is not possible to demonstrate this activity by degradation of aggrecan since the obtained CS was resolved from aggrecan and indicated a low activity of skin anti-aging effects. In general, aggrecan has a molecular mass of several million (1). It is therefore unknown whether direct absorption from the colon as a native molecule is possible. Inflammation by UV-B irradiation causes cutaneous hypertrophy (18). In addition, UV-B irradiation induces inflammatory cytokines such as interleukin-6 and TNF- $\alpha$ (19-21). Aggrecan may act with the immune system and inhibit inflammatory cytokines as an active anti-aging component.

In conclusion, findings of this study showed that oral intake of SNCEs containing abundant aggrecan may prevent skin aging. Nevertheless, more precise investigation is required to determine whether aggrecan is the active component in anti-aging in skin and to determine the exact mechanisms that prevent photoaging.

\section{Acknowledgements}

This study was supported in part by the City Area Program for the Promotion of Science and Technology in Regional Areas from the Ministry of Education, Culture, Sports, Science and Technology of Japan, and by a grant for the Promotion of International Scientific Research (2008, C-1), Japan.

\section{References}

1. Poole AA: Proteoglycans in health and disease: structures and functions. J Biol Chem 236: 1-14, 1986.

2. Iozzo RV and Murdoch AD: Proteoglycans of the extracellular environment: clues from the gene and protein side offer novel perspectives in molecular diversity and function. FASEB J 10: 598-614, 1996.

3. Hascall VC and Sajdera SW: Physical properties and polydispersity of proteoglycan from bovine nasal cartilage. J Biol Chem 245: 4920-4930, 1970.
4. Koyama Y, Hirota A, Mori H, et al: Ingestion of gelatin has differential effect on bone mineral density and body weight in protein undernutrition. J Nutr Sci Vitaminol (Tokyo) 47: 84-86, 2001.

5. Nomura Y, Oohashi K, Watanabe M and Kasugai S: Increase in bone mineral density through oral administration of shark gelatin to ovariectomized rats. Nutrition 21: 1120-1126, 2005.

6. Tanaka M, Koyama Y and Nomura Y: Effects of collagen peptide ingestion on UV-B-induced skin damage. Biosci Biotechnol Biochem 73: 930-932, 2009.

7. Kajimoto O, Odanaka W, Sakamoto W, Yoshida K and Takahashi T: Clinical effects of hyaluronic acid diet for dry skin. J New Rem Clin (in Japanese) 50: 548-560, 2001.

8. Sato T, Sakamoto W, Odanaka W, Yoshida K and Urushibara O: Clinical effects of hyaluronic acid diet for dry and rough skin. Aesthetic Dermatol 12: 109-120, 2002.

9. Mitsui T, Sashinami H, Sato F, et al: Salmon cartilage proteoglycan suppresses mouse experimental colitis through induction of Foxp3 (+) regulatory T cells. Biochem Biophys Res Commun 402: 209-215, 2010.

10. Holleran WM, Uchida Y, Halkier-Sorensen L, Haratake A, Hara M, Epstein JH and Elias PM: Structural and biochemical basis for the UVB-induced alterations in epidermal barrier function. Photodermatol Photoimmunol Photomed 13: 117-128, 1997.

11. Abe T and Mayuzumi J: The change and recovery of human skin barrier functions after ultraviolet light irradiation. Chem Pharm Bull (Tokyo) 27: 458-462, 1979.

12. Bissett DL, Hannon DP and Orr TV: An animal model of solar-aged skin: histological, physical, and visible changes in UV-irradiated hairless mouse skin. Photochem Photobiol 46: 367-378, 1987.

13. Bitter T and Muir HM: A modified uronic acid carbazole reaction. Anal Biochem 4: 330-334, 1962.

14. Abe T: Studies on skin surface barrier functions. Transepidermal water loss and skin surface lipids during childhood. Chem Pharm Bull (Tokyo) 26: 1659-1665, 1978.

15. Baker H: The effects of dimethylsulfoxide, dimethylformamide and dimethylacetamide on the cutaneous barrier to water in human skin. J Invest Dermatol 50: 283-288, 1968.

16. Iwai K, Hasegawa $\mathrm{T}$, Taguchi $\mathrm{Y}$, et al: Identification of foodderived collagen peptides in human blood after oral ingestion of gelatin hydrolysates. J Agric Food Chem 53: 6531-6536, 2005.

17. Aito-Inoue M, Lackeyram D, Fan MZ, Sato K and Mine Y: Transport of a tripeptide, Gly-Pro-Hyp, across the porcine intestinal brush-border membrane. J Pept Sci 13: 468-474, 2007.

18. Kobayashi S: UVB-induced skin damage and the protection/ treatment-effects of a novel, hydrophilic gamma-tocopherol derivative. Yakugaku Zasshi 126: 677-693, 2006.

19. Nishimura N, Tohyama C, Satoh M, Nishimura H and Reeve VE: Defective immune response and severe skin damage following UVB irradiation in interleukin-6-deficient mice. Immunology 97: 77-83, 1999.

20. Nishimura N, Reeve VE, Nishimura H, Satoh M and Tohyama C: Cutaneous metallothionein induction by ultraviolet B irradiation in interleukin-6 null mice. J Invest Dermatol 114: 343-348, 2000.

21. Osborne-Hereford AV, Rogers SW and Gahring LC: Neuronal nicotinic alpha7 receptors modulate inflammatory cytokine production in the skin following ultraviolet radiation. J Neuroimmunol 193: 130-139, 2008. 